\title{
Charge Carriers and dc Polarization Phenomena in Solid $\mathrm{Na}_{2} \mathrm{WO}_{4}$
}

\author{
P. H. BOTTELBERGHS AND E. EVERTS \\ Inorganic Chemistry Department, State University Utrecht, Croesestraat $77 a$, \\ Utrecht, The Netherlands
}

Received October 8, 1974

\begin{abstract}
Ionic transport measurements based on the Tubandt method have been performed in all three solid phases of $\mathrm{Na}_{2} \mathrm{WO}_{4}$ at temperatures between 550 and $600^{\circ} \mathrm{C}$ in air. It is shown that $t_{\mathrm{Na}}=1.00$ in all cases. $\mathrm{Ag}$ is oxidized anodically to $\mathrm{Ag}^{+}$at $\mathrm{Ag} / \mathrm{Na}_{2} \mathrm{WO}_{4}$ interfaces. Cathodically only (air) oxygen reduction occurs, resulting in the formation of a poorly conducting layer of $\mathrm{Na}_{2} \mathrm{O}$ and $\mathrm{Na}_{2} \mathrm{CO}_{3}$ at the cathode.
\end{abstract}

\section{Introduction}

In previous work we investigated the electrical conductivity of solid phases in the $\mathrm{Na}_{2} \mathrm{WO}_{4}-\mathrm{Na}_{2} \mathrm{MoO}_{4}$ system, as a function of temperature (l). $\mathrm{Na}_{2} \mathrm{WO}_{4}$ has three solid phases $(\alpha, \beta, \gamma)$ of which the two highest temperature phases $(\alpha, \beta)$ have high conductivities of the order $10^{-2} \mathrm{mho} \cdot \mathrm{cm}^{-1}$. (The $\gamma$ phase exists from ambient temperature up to $588^{\circ} \mathrm{C}$, the $\beta$-phase exists from 588 to $589^{\circ} \mathrm{C}$, and the $\alpha$-phase exists above $589^{\circ} \mathrm{C}$. The melting point is at $694^{\circ} \mathrm{C}$.)

It was then suggested that ionic conduction or, more specifically, $\mathrm{Na}^{+}$-migration was the major contribution to the conduction process, in all of the solid phases. Preliminary experiments, covering both electrolysis experiments on $\mathrm{Na}_{2} \mathrm{WO}_{4}$ and measurements of the complex admittance of $\mathrm{Na}_{2} \mathrm{WO}_{4}$ pressed pellets (with various electrode materials) as a function of sinewave frequency had given sufficient indication that in all of the solid phases, ionic migration was involved. A definite proof, however, that only $\mathrm{Na}^{+}$migration was involved still had to be given.

Tubandt methods have been applied successfully for many compounds already (2). To make an interpretation possible, in a Tubandt method the electrode reactions must be known. Chemical analysis of the anodic

and cathodic specimen constituents is necessary to deduce electrode reactions and transport numbers. Additional information about the interfacial (electrode) reactions may be obtained by transient impedance measurement methods. To date, the combined use of transient methods in solid-state electrolysis experiments with subsequent chemical analysis has not been described. In this work the advantages of combining these two measurement techniques will be exemplified for the case of determination of ionic transport numbers and electrode reactions in solid $\mathrm{Na}_{2} \mathrm{WO}_{4}$.

\section{Experimental}

\section{Sample Preparation and Mounting}

As a starting material, $\mathrm{Na}_{2} \mathrm{WO}_{4} \cdot 2 \mathrm{H}_{2} \mathrm{O}$ of high purity ("Baker Analysed") was used and was dehydrated at $220^{\circ} \mathrm{C}$ before use. The purity control and doping technique have been described in detail elsewhere (1). Very pure silver and gold disks were used as electrodes and were weighed before and after each electrolysis experiment. The cell stack consisted of three electrolyte pellets, mounted between two silver (or gold) disks. Silver thread probes of $0.01 \mathrm{~mm}$ thick were placed between the electrolyte pellets. The cell stack 
was mounted in a quartz tube cell with platinum leads. The quartz cell fitted into a quartz tube oven supplied with a Eurotherm fast cycling thyristor temperature controller.

\section{Electrical Circuitry and Techniques}

The experiments were all carried out by galvanostatic control of the overall cell current. The circuitry used for the experiments will be given in detail elsewhere. The use of chronopoteniometry to determine certain interfacial properties has been described elsewhere (3). In this work we used fast square-wave current pulses of current density $2 \times 10^{-4} \mathrm{~A} \cdot \mathrm{cm}^{-2}$ and duration $2 \times 10^{-4} \mathrm{sec}$ to determine the electrolyte resistance and the interfacial double-layer capacitance. To gather information about the electrode processes, we used slow square-wave current pulses of the same current densities but of durations up to 10 sec.

For reference we have summarized some general types of potential responses to current pulses for various cases in Fig. 1. These response types refer to so-called linear response behavior, which in principle may only occur at interfaces, when the potential difference over the interface is much smaller than $R T / n F$ (the symbols having the usual meanings). At the temperature involved here $\left(850^{\circ} \mathrm{K}\right)$, it means that one may expect a linear response behavior to occur for a one-electron process when the overpotential is much smaller than $73 \mathrm{mV}$. (A two-electron process obviously involves half this value.)

During the electrolysis with a $1 \mathrm{~mA}$ current, the potential differences of anode and cathode with respect to the probes were recorded simultaneously. At appropriate time intervals fast and slow-pulse response were recorded oscillographically for both electrodes.

\section{Chemical Analysis}

After each electrolysis experiment, the cell stack was sectioned into its three original parts and the anodic and cathodic electrolyte pellets were analyzed chemically.

The anodic pellet was brought into $50 \mathrm{ml}$ of distilled water and then hot-stirred in the dark with dropwise addition of ammonia

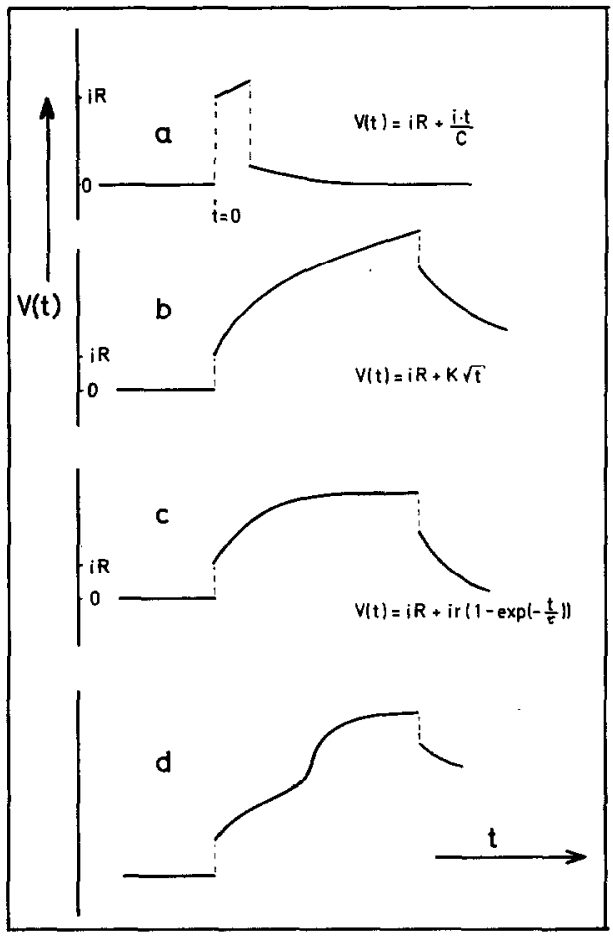

FIG. 1. Some general types of potential-time curves obtained in transient impedance measurements with the galvanostatic pulse technique. (a) Fast pulse response of duration of the order $0.2 \mathrm{msec}$; electrolyte resistance and double layer capacitance may be derived directly from this response. (b) Semiparabolic response obtained with slow current pulses (1-10 sec) when diffusional processes are involved at the interface. (c) Exponential response, observed when the electrode reactions are charge-transfer controlled. (d) Twoprocessed response due to depletion of the primarily reacting electroactive species at the interface.

until all the $\mathrm{Ag}_{2} \mathrm{WO}_{4}$ had dissolved $\left(\mathrm{Na}_{2} \mathrm{WO}_{4}\right.$ dissolves readily). Next the solution was titrated potentiometrically with a standardized KI-solution. A very clean $\mathrm{Ag}$ wire $1 \mathrm{~mm}$ thick was used as a measuring electrode, and a $\mathrm{Ni} / \mathrm{NiSO}_{4}$-electrode (saturated $\mathrm{NiSO}_{4}$ solution) was used as a reference electrode with a Keithly 602 electrometer. The cathodic pellet was dissolved in $100 \mathrm{ml}$ of distilled water. To $50 \mathrm{ml}$ of this solution, excess $\mathrm{BaCl}_{2}$ solution was added to precipitate all the tungstate and carbonate. Next the solution was titrated potentiometrically (using an Ingold combined glass electrode and a Metrohm 
E350B pH meter) with a standardized $\mathrm{HNO}_{3}$ solution, thus giving the amount of $\mathrm{OH}^{-}$ The other $50 \mathrm{ml}$ was titrated directly with $\mathrm{HNO}_{3}$ down to $\mathrm{pH} \sim 7$, thus giving the summed amount of $\mathrm{OH}^{-}$and $\mathrm{CO}^{2-}$; in this case the equivalence point $\left(\mathrm{HCO}_{3}{ }^{-}\right)$was found at a $\mathrm{pH}$ of about 8.4 (the $\mathrm{pH}$ meter, however, was not calibrated exactly). Thus, the total amount of originally formed $\mathrm{Na}_{2} \mathrm{O}$ at the cathode (giving the Faraday equivalent in the electrolysis) and the amount of $\mathrm{Na}_{2} \mathrm{CO}_{3}$ (formed from the oxide and atmospheric $\mathrm{CO}_{2}$ at the high temperature), can be found.

\section{Results}

In all cases, the slow and fast pulse responses of the cell stack were measured before the electrolysis was started. Also, the open-cell voltages between the probes and the working electrodes were measured. In most cases, the electrolyte resistance of a pellet of dimensions: $\phi=0.50 \mathrm{~cm}^{2}$ and $\mathrm{d}=0.3 \mathrm{~cm}$ was of the order of $50 \mathrm{ohm}$ at approximately $600^{\circ} \mathrm{C}$.

The open-cell voltages depended greatly on the electrode materials used. A silver disk electrode measured against a silver probe gave an EMF of less than $20 \mathrm{mV}$ (whereas Pt-paint measured against a silver probe ranged from $100-300 \mathrm{mV}$ ).

\section{Electrolyte Resistance}

Using fast current pulses, the electrolyte resistance of all three pellets was measured during the electrolysis (at $1 \mathrm{~mA}$ electrolysis current). The results are summarized in Table I, for a typical experiment. Although there are some variations in the anodic and middle pellets, which may be due to a number of causes, the increase in electrolyte resistance of the cathodic pellet is much more pronounced and proceeds with time. This is to be expected, as will be discussed subsequently.

\section{Overall Cell Polarization}

A typical example of observed Warburg (diffusional) impedance behavior at $\mathrm{Ag} / \mathrm{Na}_{2}$ $\mathrm{WO}_{4}$ interfaces before electrolysis is reflected in Fig. 2 where we have plotted $V(t)-i R_{e}$ against $(t)^{1 / 2}$. The data were found by calculation from oscillographic recordings. It may seem odd that real Warburg (diffusional) impedance behavior occurs before electrolyzing, as there is not supposed to be any $\mathrm{Ag}^{+}$present in the electrolyte. If this were true, the starting potential would not be defined first of all, and furthermore, since the bulk silver ion concentration in the Nernst formula for the overpotential would be zero, the logarithmic dependence of the potential as a function of the silver ion concentration at the interface might not be linearized. However, we have strong evidence that a silver electrode in contact with solid $\mathrm{Na}_{2} \mathrm{WO}_{4}$ in the $\alpha$-phase (at $600^{\circ} \mathrm{C}$ ) undergoes a fairly rapid, minor degree of oxidation in air, resulting in the formation of $\mathrm{Ag}_{2} \mathrm{O}$ dissolved in the tungstate. This process may occur within several hours, giving an $\mathrm{Ag}^{+}$concentration of about $0.1 \mathrm{~mole} \%$ near the interface. This also

TABLE I

Electrolyte Resistances in the Cell Stack with Silver Anode and Cathode During Electrolysis with Constant Current of $1 \mathrm{~mA}^{a}$

\begin{tabular}{|c|c|c|c|c|c|c|c|c|}
\hline \multirow[b]{2}{*}{ Resistance between: } & \multicolumn{8}{|c|}{ Time, in minutes } \\
\hline & 0 & 63 & 150 & 195 & 215 & 255 & 335 & 390 \\
\hline Anode and probe 1 & 53 & 53 & 54 & 60 & & 60 & 49 & 49 \\
\hline Probe 1 and probe 2 & 54 & 56 & 59 & 50 & & & 60 & 62 \\
\hline Cathode and probe 2 & 54 & 84 & 84 & 600 & 400 & 560 & 880 & 4400 \\
\hline
\end{tabular}

a Current density is $2 \mathrm{~mA} \cdot \mathrm{cm}^{-2}$. Resistances are measured using fast current pulses and are given in ohms. 


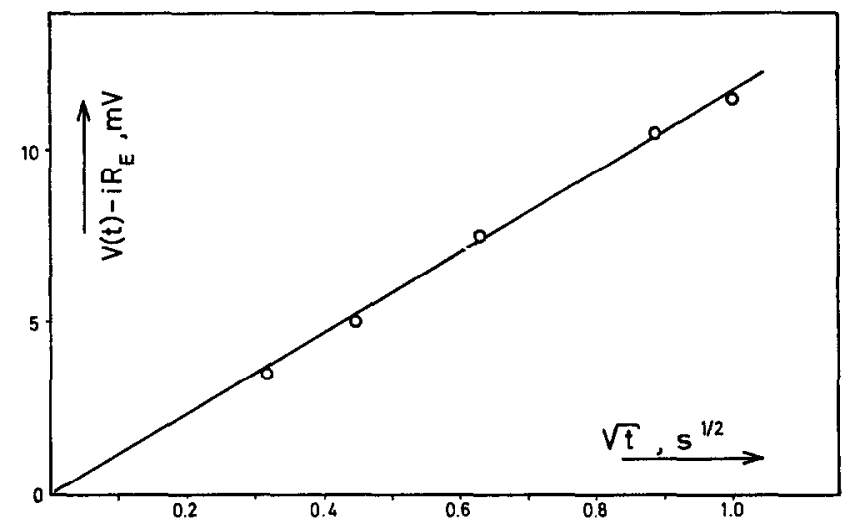

FIG. 2. Overpotential behavior of $\mathrm{Ag} / \mathrm{Na}_{2} \mathrm{WO}_{4}$ interface plotted against $(\mathrm{t})^{1 / 2}$. This type of behavior refers to the transient depicted in Fig. 1 b.

explains why a silver thread may be used as a potential probe. With other compounds this may not be the case, and thus, this method may not be applied as such.

The simultaneous recording of both the overall potential difference anode-probe or cathode-probe and the corresponding electrolyte resistance (by fast pulses) enables a better understanding of certain polarization effects, occurring during the electrolysis. This is shown in Fig. 3, where we have plotted the potential difference between the cathode and the adjacent probe versus time. In the same plot the ohmic drop is shown as it was calculated from fast pulse responses. Obviously, interfacial polarization effects contribute mainly to the overall polarization in Fig. 3 in the beginning of the electrolysis: $V(t)$ rises rapidly with time whereas $R_{e}$ remains almost constant. After $2 \frac{1}{2} \mathrm{hr}$, there is a marked increase in $i R_{e}$, but the increase in $V(t)$ is even larger. This means that the increase in $V(t)$ must be assigned to the formation of a new electrolyte layer (which conducts less than $\left.\mathrm{Na}_{2} \mathrm{WO}_{4}\right)$ as much as to an increased polarization at this new interface. The subsequent decrease in $V(t)$ at $t \sim 220$ min may be attributed entirely to a decrease in the electrolyte resistance. After $6 \mathrm{hr}$, the electrolyte resistance increases considerably, thus determining the overall polarization completely. For the cathodic potential difference there is a

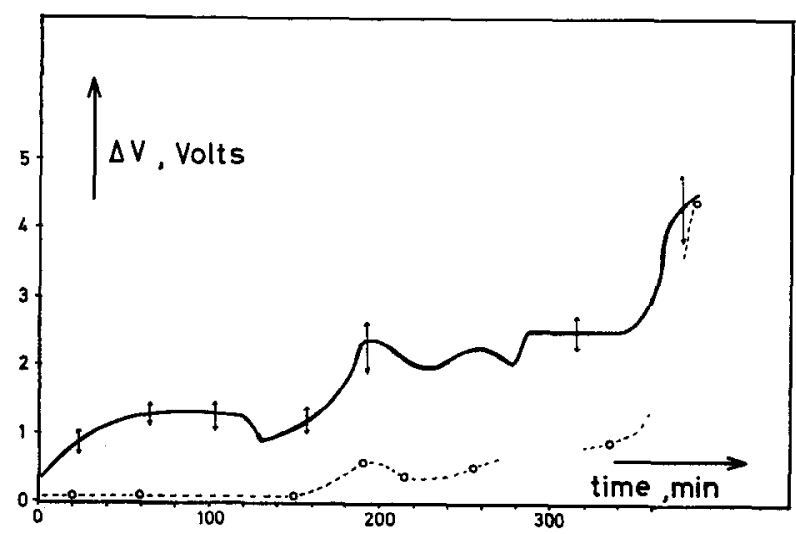

Fig. 3. Potential difference between cathode and probe 2 during electrolysis (solid line). Broken line interconnects the calculated values of the ohmic drop over the electrolyte, as obtained from fast current pulses (open circles). The instability in the overall potential difference is depicted by thin vertical arrows. 
great deal of instability in the signal, as depicted in Fig. 3. This may not merely be assigned to pick-up at the electrolyte resistance, although it may well be of influence. The anodic potential difference is not shown for the same experiment, since it showed very little time dependent polarization after some $40 \mathrm{~min}$, in fair agreement with the $R_{e}$ behavior of the anodic pellet. In the electrolysis with a silver anode, after some time there is no more variation of the anodic potential (measured against its adjacent probe). This implies that the chemical activity of the electroactive species becomes constant after some time, which agrees with the results of the slow pulse response studies at the silver anode. No interfacial polarization is seen with anodic current pulses of duration up to say $10 \mathrm{sec}$.

The use of reversed (cathodic) pulses of high current density might show polarization effects due to depletion of $\mathrm{Ag}^{+}$at the anode, but these were not studied here. On the other hand, cathodic polarization remains quite marked during the whole experiment. This may be reflected in Fig. 4, where slow pulse responses are shown for the overall impedance between the cathode and its adjacent probe.

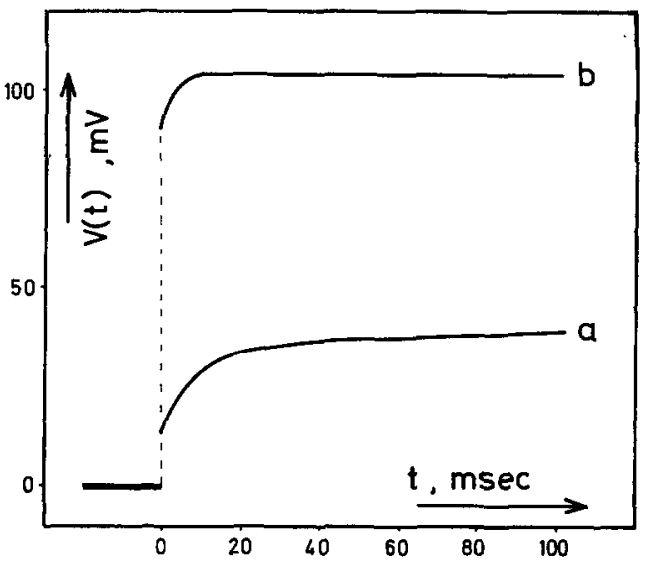

FIG. 4. Typical transients for cathodic interface $\mathrm{Ag} / \mathrm{Na}_{2} \mathrm{WO}_{4}$. (a) After $110 \mathrm{~min}$ electrolysis time (experiment 740424). (b) After $270 \mathrm{~min}$ electrolysis time (same experiment).

In Fig. 4b we may have the case of a chargetransfer controlled interfacial process. This applies to the silver cathode in contact with the new electrolyte.

\section{Chemical Analysis}

The results of the chemical analysis for a number of electrolysis experiments are shown

\section{TABLE II}

Results of Chemical analysis of Anodic and Cathodic Electrolyte Pellets and of Anodic Weight Decrease after Galvanostatic Electrolysis (AT $1 \mathrm{~mA}$ ) of Solid $\mathrm{Na}_{2} \mathrm{WO}_{4}{ }^{a}$

\begin{tabular}{|c|c|c|c|c|c|}
\hline $\begin{array}{c}\text { Experiment } \\
\text { number }\end{array}$ & Phase & $\begin{array}{l}\text { Passed charge } \\
\text { in } \\
\text { milliFaradays }\end{array}$ & $\begin{array}{l}\text { Anodic weight } \\
\text { decrease, in } \\
\text { millimoles }\end{array}$ & $\begin{array}{l}\mathrm{Ag}^{+} \text {, amount in } \\
\text { anodic pellet, } \\
\text { in millimoles }\end{array}$ & $\begin{array}{l}\mathrm{OH}^{-}+\mathrm{CO}_{3}^{2-} \text {, total } \\
\text { amount of base } \\
\text { in cathodic pellet, } \\
\text { in milliequivalents }\end{array}$ \\
\hline 740321 & $\alpha$ & 0.213 & 0.213 & 0.212 & ${ }^{b}$ \\
\hline 740326 & $\alpha$ & 0.206 & 0.222 & 0.219 & $-^{b}$ \\
\hline 740424 & $\alpha$ & 0.252 & 0.233 & $0.199^{c}$ & $\ldots$ \\
\hline 740506 & $\gamma$ & 0.055 & 0.044 & $0.033^{c}$ & 0.054 \\
\hline 740515 & $\gamma$ & 0.22 & - & - & $0.29^{d}$ \\
\hline 740530 & $\alpha$ & 0.236 & 0.246 & 0.232 & $0.268^{d}$ \\
\hline 740604 & $\beta$ & 0.272 & 0.267 & 0.207 & 0.268 \\
\hline 740605 & $\alpha$ & 0.284 & 0.288 & 0.267 & 0.281 \\
\hline
\end{tabular}

a All experiments in this table were carried out with silver anodes and cathodes, except 740515 , where a gold anode was used.

${ }^{b}$ Improper determination of base amount.

c Ag electrode not well cleansed after sectioning.

d Value too high due to unknown cause. 
in Table II. Apart from the experiments gathered in this table, we performed some more electrolysis experiments. In these we did not collect all possible data, but they gave some important additional information. One important fact to be mentioned is the difference in the results of electrolysis experiments with gold anodes in the $\alpha$ and $\gamma$-phases of $\mathrm{Na}_{2} \mathrm{WO}_{4}$. In the experiment at the $\gamma$-phase, a yellow layer was formed at the anode, which was not the case in the $\alpha$-phase. The origin of this phenomenon will be discussed.

\section{Discussion}

\section{Anodic Processes}

From the results of the chemical analysis after the electrolysis experiments, the electrode reactions can be deduced. When silver is used as anode material, the following reaction occurs:

$$
\mathrm{Ag} \rightarrow \mathrm{Ag}^{+}+\mathrm{e}
$$

When the total amount of $\mathrm{Ag}^{+}$is still small, we might state that $\mathrm{Ag}^{+}$ions formed at the anode, will occupy cation sites in the pure $\mathrm{Na}_{2} \mathrm{WO}_{4}$ structure, thus giving rise to the formation of defects $\operatorname{Ag}_{\mathrm{Na}}^{x}$. However, when the total amount of $\mathrm{Ag}^{+}$ions becomes larger at progression of the electrolysis, it becomes doubtful to what extent one may still speak about defects such as $\mathrm{Ag}_{\mathrm{Na}}^{x}$. In a far progressed stage it might be more appropriate to define $\mathrm{Na}_{\mathbf{A g}}^{x}$ defects in an $\mathrm{Ag}_{2} \mathrm{WO}_{4}$ structure. Furthermore, one should consider the possibility of phase segregation and even of eutectic melting. Thus, an examination of the phase diagram involved in the formation of compounds or mixed crystals during the electrolysis should always be included.

In this case, the phase diagram of the $\mathrm{Na}_{2} \mathrm{WO}_{4}-\mathrm{Ag}_{2} \mathrm{WO}_{4}$ system is thus of importance. An extensive treatment of the phase diagram (4) and the diffusional problems (5) will be given elsewhere. Based on theoretical considerations, it can be shown that when using an electrolysis current density of 2 $\mathrm{mA} \cdot \mathrm{cm}^{-2}$ for $8 \mathrm{hr}$ or less, no (local) eutectic melting will occur in the $\mathrm{Ag}_{2} \mathrm{WO}_{4}-\mathrm{Na}_{2} \mathrm{WO}_{4}$ system at $600^{\circ} \mathrm{C}(5)$. The phase diagram of
$\mathrm{Na}_{2} \mathrm{WO}_{4}-\mathrm{Ag}_{2} \mathrm{WO}_{4}$ is rather involved (4). Above 45 mole \% it is doubtful to what extent one may still speak of an $\alpha$-phase-even phase segregation may be possible. The occurrence of the $\beta$-phase is even more critical: above 3 mole $\% \mathrm{Ag}_{2} \mathrm{WO}_{4}$ no $\beta$-phase exists. Experiments in the $\gamma$-phase present less problems in this respect, but they are of little interest since we are mainly concerned with highly conducting phases.

Electrolysis experiments in the $\alpha$-phase are best performed at temperatures between 590 and $600^{\circ} \mathrm{C}$ in view of the possibility of local melting. When electrolysis is performed with a gold anode, the results are quite different. Gold is completely inert as an electrode material. A thermodynamically acceptable electrode reaction is:

$$
\mathrm{WO}_{4}^{2-} \rightarrow \mathrm{WO}_{3}+\frac{1}{2} \mathrm{O}_{2}+2 \mathrm{e}
$$

In the $\gamma$-phase the $\mathrm{WO}_{3}$ is observed to be formed as a separate layer between the anode and the electrolyte. As this is not observed with the same experiment in the $\alpha$-phase, the $\mathrm{WO}_{3}$ probably may then react with the tungstate, to give polytungstates, which are colorless.

\section{Cathodic Processes}

At the cathode, the following reduction reaction occurs:

$$
\frac{1}{2} \mathrm{O}_{2}+2 \mathrm{e} \rightarrow \mathrm{O}^{2-}
$$

as can be deduced from the fact that the Faraday amount of base is found in the cathodic pellets. This also implies that no oxygen ion migration is involved in the electrolytic transport processes because in that case a smaller amount of base should be found in the cathodic pellet.

One might consider the possibility that tungstate is reduced at the cathode. However, this is not the case, as is evidenced from the fact that the cathodic pellet was always colorless, while lower tungsten oxides would give a colored product. The formation of lower tungsten oxides as a cathodic process may be expected to occur when the electrolysis is performed in a pure $\mathrm{N}_{2}$ atmosphere, involving a sufficiently low oxygen partial pressure. The diffusion rate of $\mathrm{O}^{2-}$ ions in the tungstate is 
probably very low. Thus, it is inevitable that after some electrolysis time, a layer of $\mathrm{Na}_{2} \mathrm{O}$ is segregated at the cathode. In the presence of $\mathrm{CO}_{2}$ a combined product may be formed: $\left(\mathrm{Na}_{2} \mathrm{O}\right)_{a}\left(\mathrm{Na}_{2} \mathrm{CO}_{3}\right)$. By use of the differential base-equivalent titration method, as described in the experimental section, we found: $a \sim 0.05-0.3$. However, this value may differ markedly in different circumstances. The gradual formation of an oxide/carbonate layer is reflected in Fig. 3.

The instability of the measured overall voltage difference over the cathodic pellet may well be due to instability in the current density distribution at the cathodic interface. Locally the formation of poorly conducting layers will lead to a continuously changing current density distribution pattern, giving a messy overall picture. It should be stressed that as opposed to the cathodic potential difference behavior, the anodic potential difference shows no instability whatsoever (in those cases where an $\mathrm{Ag}$ electrode was used). The marked difference in the slow pulse response behavior at the cathode as demonstrated by Fig. 4 may be attributed to a depletion of the original electroactive species $\mathrm{Ag}^{+}$in the case of a silver cathode). The processes at the cathode (Fig. 4b) are then determined by an oxygen process such as:

$$
\mathrm{O}^{2-} \underset{\text { cathodic }}{\stackrel{\text { anodic }}{\rightleftharpoons}} \frac{1}{2} \mathrm{O}_{2}+2 \mathrm{e}
$$

These processes may give rise to an activation polarization.

\section{Transport Numbers}

First, it should be stressed that in the electrolysis experiments as they have been performed, systematic errors are easily introduced by improper sectioning of the cell stack or by insufficient dissolving of the electrolyte pellets, as only very small quantities are involved. One may get some impression of these deviations from Table II. Nevertheless, the overall results are very satisfactory and emphasize the previously mentioned expectation that the transport number in all three phases of $\mathrm{Na}_{2} \mathrm{WO}_{4}$ is $t_{\mathrm{Na}^{+}}=1.00$. This conclusion can be drawn in accordance with the electrode reactions which already have been discussed.

\section{Conclusion}

From the results of the chemical analysis, performed after over nine electrolysis experiments, it may be concluded that in all of the solid phases of $\mathrm{Na}_{2} \mathrm{WO}_{4}, t_{\mathrm{Na}^{+}}=1.00$. A statistical evaluation of the accuracy is not appropriate for this result since the main error sources introduce systematic rather than random errors. When a silver anode is used, the electrode reaction is: $\mathrm{Ag} \rightarrow \mathrm{Ag}^{+}+\mathrm{e}$. With a gold anode, the reaction is: $\mathrm{WO}^{2-} \rightarrow \mathrm{WO}_{3}+$ $\frac{1}{2} \mathrm{O}_{2}+2 \mathrm{e}$.

Cathodic reactions seem to be only slightly dependent on the electrode material used and involve reduction of (air) oxygen, giving rise to the formation of poorly conducting layers of $\mathrm{Na}_{2} \mathrm{O}$ and $\mathrm{Na}_{2} \mathrm{CO}_{3}$ at the cathode. It should be stressed (with special reference to Fig. 3) that the method described here, is a valuable tool for studying solid electrolytes under operating conditions, e.g., in galvanic cells.

\section{Acknowledgments}

The authors are indebted to Dr. G. H. J. Broers for many stimulating discussions and for his reading of the manuscript.

\section{References}

1. P. H. Bottelberghs and F. R. van Buren, $J$. Solid State Chem. 13, 182 (1975).

2. C. Tubandt, "Handbuch der Experimentalphysik," Vol. 12, no. 1, p. 383, 1932.

3. J. HLADIK (Ed), "Physics of Electrolytes," Vol. 2, p. 567, 1973, Academic Press, New York.

4. P. H. BotTELBERoHS AND E. EVERTs, to be published.

5. P. H. Bottelberghs and G. H. J. Broers, to be published. 